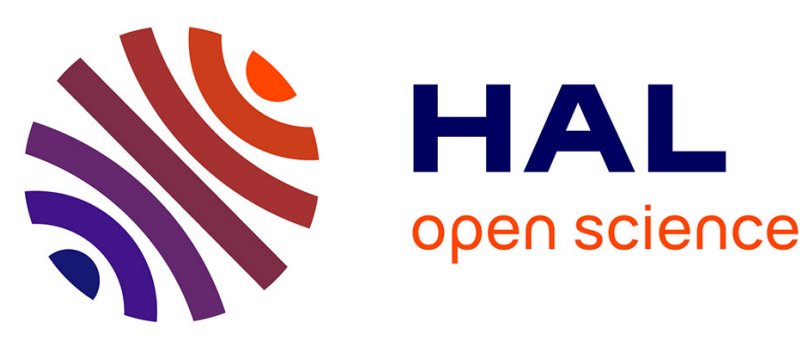

\title{
Modeling somite scaling in small embryos in the framework of Turing patterns
}

Laurence Signon, Bogdan Nowakowski, Annie Lemarchand

\section{To cite this version:}

Laurence Signon, Bogdan Nowakowski, Annie Lemarchand. Modeling somite scaling in small embryos in the framework of Turing patterns. Physical Review E , 2016, 93 (4), pp.042402 10.1103/PhysRevE.93.042402 . hal-01314303

\section{HAL Id: hal-01314303 https://hal.sorbonne-universite.fr/hal-01314303}

Submitted on 11 May 2016

HAL is a multi-disciplinary open access archive for the deposit and dissemination of scientific research documents, whether they are published or not. The documents may come from teaching and research institutions in France or abroad, or from public or private research centers.
L'archive ouverte pluridisciplinaire HAL, est destinée au dépôt et à la diffusion de documents scientifiques de niveau recherche, publiés ou non, émanant des établissements d'enseignement et de recherche français ou étrangers, des laboratoires publics ou privés. 


\section{Modelling somite scaling in small embryos in the framework of Turing patterns}

Laurence Signon $^{1,2}$, Bogdan Nowakowski ${ }^{3,4}$ and Annie Lemarchand ${ }^{5,6}$

1 Université Paris-Sud, Institut de génétique et microbiologie,

15, rue Georges Clémenceau, 91405 Orsay cedex, France

2 CNRS UMR 8621, IGM, Orsay, France

3 Institute of Physical Chemistry, Polish Academy of Sciences,

Kasprzaka 44/52, 01-224 Warsaw, Poland

4 SGGW, Warsaw University of Life Sciences,

Nowoursynowska 159, 02-776, Warsaw, Poland

5 Sorbonne Universités, UPMC Univ Paris 06, UMR 7600,

Laboratoire de Physique Théorique de la Matière Condensée,

4, place Jussieu, case courrier 121, 75252 Paris Cedex 05, France

6 CNRS, UMR 7600 LPTMC, Paris, France

January 25, 2016

Corresponding author: Annie Lemarchand, E-mail: anle@lptmc.jussieu.fr, Phone: 331442744 55, Fax: 33144275100 


\begin{abstract}
The adaptation of prevertebra size to embryo size is investigated in the framework of a reaction-diffusion model involving a Turing pattern. The reaction scheme and Fick's first law of diffusion are modified in order to take into account the departure from dilute conditions induced by confinement in smaller embryos. In agreement with the experimental observations of scaling in somitogenesis, our model predicts the formation of smaller prevertebrae or somites in smaller embryos. These results suggest that models based on Turing patterns cannot be automatically disregarded by invoking the question of maintaining proportions in embryonic development. Our approach highlights the non trivial role that the solvent can play in biology.
\end{abstract}

PACS: 87.17.Pq, 82.40.Ck, 82.20.Yn, 87.18.Vf

\title{
1 INTRODUCTION
}

Scaling of pattern formation with embryo size is a universal feature observed in many organisms and the question of maintaining proportions is relevant for both vertebrates $[1,2,3]$ and invertebrates $[4,5,6,7]$. However, the formation of patterns that are proportional to the size of the embryos remains a poorly understood property of development. Morphogen gradient is a widely accepted feature by which a developing tissue provides its cells with positional information [8,9]. The ability of an embryo to adapt to size variations is often related to the scaling of morphogen gradient with global embryo size $[2,5,6,7]$. We recently postulated that a reactiondiffusion model based on a Turing pattern could account for prevertebra or somite formation and non trivial experiments have been reproduced $[10,11,12,13]$. In order to further investigate the validity of the model, we wish to examine if it could account for scaling of pattern formation.

Since the observation of temporal oscillations of some morphogens in the undifferentiated tissue or presomitic mesoderm [14], the clock and wavefront model [15] has been the most commonly admitted model of somitogenesis [16, 17, 18, 19]. Nevertheless, this model has been lately challenged by recent experiments that show the formation of somites without the need of gene oscillations and clocks [20]. As an alternative to clock and wavefront type models, reaction-diffusion processes offer a minimal framework to model the formation of somites without losing the molecular scale. In this context, a Turing structure, i.e. a spatially-periodic oscillation of morphogen concentrations is supposed to develop behind a propagating chemical wave front. This prepattern is then admitted to induce a complex cascade of pathways, eventually leading to the differentiation of tissues and vertebra formation [21, 22, 23, 24, 25, 26]. However, the connection between spine development and Turing instability remains a matter of debate [27]. The main criticism against Turing pattern is that it does not a priori account for scaling of patterning and size adaptation of the somites to the global size of an embryo. Indeed, the wavelength of a Turing structure is fixed by dynamics, the rate constants of the reactions and the 
diffusion coefficients of the chemical species, and not by system size [28]. Different ways to preserve proportion in Turing pattern have been achieved by introducing size-dependent dynamical parameters or additional species whose concentration depends on system size $[29,30,31,32]$.

Furthermore, physiological media are known to suffer from confinement [33, 34, $35,36,37,38]$ and we propose to address the issue of size adaptation in the general context of molecular crowding. We start from the intuitive statement that the effect of confinement is stronger in smaller embryos. In these conditions, the usual assumptions about dilute solutions may fail and the role of water or solvent in the chemical scheme may not be ignored [39]. Consequently, the rate constants [33, 34, 40] and the diffusion coefficients [41] may be both modified. More precisely, we propose to incorporate the solvent in the chemical scheme and to examine the deviation to usual Fick's law of diffusion in the framework of linear irreversible thermodynamics $[42,43]$. The effects of concentration-dependent diffusivity and enhancement of the concentration of some reactant on Turing patterns have been extensively investigated $[44,45,46,47,48]$. Specifically, our aim is to determine whether a strengthening of confinement may induce a decrease of structure wavelength and consequently somite size in smaller embryos.

The paper is organized as follows. In section 2, we present the reaction-diffusion model and the modified partial differential equations in the presence of confinement. The numerical integration procedure is made precise in section 3 . The results are discussed in section 4 . Section 5 is devoted to conclusion. The detailed derivation of the modified laws of diffusion in the presence of a departure from ideality is given in the appendix A. The appendix B contains an analytical derivation of the perturbed wavelength of the spatial structure in the limit where the effect of confinement is stronger on reaction than diffusion.

\section{MODEL: REACTION-DIFFUSION EQUATIONS IN A CROWDED ENVIRONMENT}

We recently studied the following reaction scheme, inspired from the Schnakenberg model [49] and the Gray-Scott model [50], to account for the formation of somites 
$[10,11,12,13]:$

$$
\begin{array}{rcr} 
& \stackrel{k_{1}}{\mathrm{~A}} & \\
& \rightarrow & \mathrm{R}_{1} \\
& & \\
& \stackrel{k_{2}}{\mathrm{~A}}+\mathrm{B} & \\
& \rightarrow & 3 \mathrm{~A} \\
\mathrm{~B} & \stackrel{k_{3}}{\rightleftharpoons} & \\
& \mathrm{R}_{2} \\
k_{-3}^{\prime} &
\end{array}
$$

where the densities of species $R_{1}$ and $R_{2}$ are kept constant due to appropriate exchanges with reservoirs. Species A, expressed at the rostral end of the embryo, may be identified with retinoic acid (RA) and genes involved in RA signaling, whereas species B, present at the caudal end, may been related to the fibroblast growth factor (Fgf) and genes involved in Fgf pathway [51]. Different couples of antagonist gradients are found in the literature and may play the role of the activator $\mathrm{A}$ and the inhibitor B of the Turing structure, provided that B diffuses faster than species $\mathrm{A}[18,52]$. In the absence of a perturbation, the dynamics of the densities $\rho_{A}$ and $\rho_{B}$ of species $\mathrm{A}$ and $\mathrm{B}$ is governed by the following reaction-diffusion equations:

$$
\begin{aligned}
& \frac{\partial \rho_{A}}{\partial t}=-k_{1} \rho_{A}+k_{2} \rho_{A}^{2} \rho_{B}+D_{A}^{*} \frac{\partial^{2} \rho_{A}}{\partial x^{2}} \\
& \frac{\partial \rho_{B}}{\partial t}=k_{-3}-k_{3} \rho_{B}-k_{2} \rho_{A}^{2} \rho_{B}+D_{B}^{*} \frac{\partial^{2} \rho_{B}}{\partial x^{2}}
\end{aligned}
$$

where $k_{i},(i=1,2,3,-3)$, are rate constants, $D_{A}^{*}$ and $D_{B}^{*}$ are the diffusion coefficients of species $\mathrm{A}$ and $\mathrm{B}$, respectively. We have set $k_{-3}=k_{-3}^{\prime} \rho_{R_{2}}$ for the apparent rate constant of the reverse step of Eq. (3). Provided that species B diffuses faster than species A and for well-chosen rate constant values, a Turing pattern develops. We focussed on the beginning of growth and did not consider the termination process of somite formation $[10,12,13]$. The reaction-diffusion system has been shown to correctly mimic the alteration of the structure wavelength due to the introduction of a local source of $\mathrm{B}$ in the undifferentiated tissue $[12,13,16,53]$. However, the ability of Turing structures to reproduce the fact that smaller embryos of a same animal species show the same number of somites of smaller size, remains under debate.

To investigate this issue, we admit that the smaller size of an embryo may induce spatial crowding and a departure from the usual assumptions about dilute solutions. Indeed, both retinoic acid and Fgf signaling depend on maternal factors $[54,55,56]$ and it is reasonable to assume that the concentration of maternal factors is higher in smaller embryos. Interestingly, the concentration of another growth-promoting factor, the insulin-like growth factor-I, has been reported to be higher in smaller embryos of pigs [57]. Hence, the smaller size of an embryo leads to a strengthening of confinement. Then, the hypothesis according to which the solvent is in great 
excess and does not need to be taken into account in the reaction scheme may not be valid [43]. To be explicit, we introduce the following interactions between the reactive species and the solvent. We make the hypothesis that the supply of species $\mathrm{B}$ from the reservoir $\mathrm{R}_{2}$ simultaneously drops the solvent into the surroundings. Conversely, the elimination of $\mathrm{A}$ and $\mathrm{B}$ through exchanges with the reservoirs is supposed to require an interaction with the solvent. In the case of a dilute solution of species $\mathrm{A}$ and $\mathrm{B}$, the role of the solvent is hidden in the reaction mechanism given in Eqs. (1-3) but, when the solution is not dilute enough for the density of the solvent to remain constant, the schemes of supply and removal processes of species $\mathrm{A}$ and $\mathrm{B}$ is completed as follows:

$$
\begin{array}{ccc}
\mathrm{A}+* & \stackrel{k_{1}^{*}}{\rightarrow} & \mathrm{R}_{1} \\
& & \\
2 \mathrm{~A}+\mathrm{B} & \stackrel{k_{2}}{\rightarrow} & \\
& & \\
& \stackrel{k_{3}^{*}}{\rightleftharpoons} & \\
\mathrm{B}+* & \mathrm{R}_{2} \\
& k_{-3}^{\prime} &
\end{array}
$$

where $*$ denotes a particle of the solvent and the autocatalytic step given in Eq. (2) is unchanged. Hence, the density of the solvent, $\rho_{*}$, is likely to vary. The balance equation for the density $\rho_{I}$ of species I reads:

$$
\frac{\partial \rho_{I}}{\partial t}+\vec{\nabla} \cdot \vec{j}_{I}=\left.\frac{\partial \rho_{I}}{\partial t}\right|_{\text {reac }}, \quad I=A, B, *
$$

where the right-hand side accounts for the variation of $\rho_{I}$ in the volume due to reactions and the flux $\vec{j}_{I}$ is the mass flow of species I through a unit surface per unit time. The exchanges with the reservoirs of $R_{1}$ and $R_{2}$ guarantee that the fluxes $\vec{j}_{R_{1}}$ and $\vec{j}_{R_{2}}$ exactly compensate the reactive terms $\left.\frac{\partial \rho_{R_{1}}}{\partial t}\right|_{\text {reac }}$ and $\left.\frac{\partial \rho_{R_{2}}}{\partial t}\right|_{\text {reac }}$, respectively, so that $\rho_{R_{1}}$ and $\rho_{R_{2}}$ remain constant. Confinement is not supposed to interfere with the performances of the reservoirs of chemical species. We admit that the so-called chemostats are able to impose that the densities $\rho_{R_{1}}$ and $\rho_{R_{2}}$ of species $R_{1}$ and $R_{2}$ remain homogeneous and constant. In the following, the total density is defined as:

$$
\rho=\rho_{A}(x, t)+\rho_{B}(x, t)+\rho_{*}(x, t)
$$

in which the invariant densities of species $R_{1}$ and $R_{2}$ can be disregarded. The flux obeys

$$
\vec{j}_{I}=\rho_{I}\left(\vec{u}_{I}-\vec{u}\right)
$$

where $\vec{u}_{I}$ is the velocity of the center of mass of species I, and $\vec{u}=\frac{1}{\rho} \sum_{I=A, B, *} \rho_{I} \vec{u}_{I}$, the velocity of the center of mass of the fluid. Using Eq. (10) for the total density 
and the definition of the velocity of the center of mass, we immediately show that the sum of all the fluxes vanishes:

$$
\vec{j}_{A}+\vec{j}_{B}+\vec{j}_{*}=0 .
$$

Then, summing Eq. (9) over $I=A, B$, * and taking Eq. (12) into account, we get

$$
\frac{\partial \rho}{\partial t}=\left.\sum_{I=A, B, *} \frac{\partial \rho_{I}}{\partial t}\right|_{\text {reac }}
$$

In the absence of nuclear reactions, the right-hand side vanishes due to the conservation of the total mass, so that the total density $\rho$ is constant. A solution of species $\mathrm{A}$ and $\mathrm{B}$ is said to be dilute if $\rho_{A}(x, t) / \rho$ and $\rho_{B}(x, t) / \rho$ tend to zero, which amounts to neglect the variations of the density of the solvent $\rho_{*}(x, t)$. In these conditions, the two chemical schemes given in Eqs. (1-3) and Eqs. (6-8) are identical.

If the surroundings are crowded and the solution cannot be considered sufficiently dilute, the laws of diffusion may be modified. Diffusion typically enters into the class of phenomena that are described in the framework of linear irreversible thermodynamics [42]. According to the second law of thermodynamics, the entropy production is a positive quantity that can be written as the sum of products of thermodynamic fluxes and forces. In the linear domain of irreversible thermodynamics, these fluxes and forces are linked by linear relationships. Fick's first law is an example of such a linear relationship between a flux of matter and the conjugated thermodynamic force. In the case of an ideal solution and for reasonable assumptions, the diffusion of a chemical species only depends on this species. In a non dilute solution, the linear relationships between the fluxes and the conjugated forces of all the species imply that the diffusion of a species depends on the densities and the diffusion coefficients of the other species. The resulting Fick's first law, valid in a non dilute solution, is derived in the appendix A.

Finally, the reaction-diffusion equations governing the evolution of the densities of species A and B engaged in the chemical scheme given in Eqs. (6-8) are deduced from the balance equation given in Eq. (9) and the Fick's first law given in Eqs. (A.13,A.14). It reads:

$$
\begin{aligned}
\frac{\partial \rho_{A}}{\partial t}= & -k_{1} \frac{\rho_{A}}{\rho}\left(\rho-\rho_{A}-\rho_{B}\right)+k_{2}\left(\rho_{A}\right)^{2} \rho_{B} \\
& +D_{A}^{*}\left[\left(1-\frac{\rho_{A}}{\rho}\right) \Delta \rho_{A}-\frac{\left(\vec{\nabla} \rho_{A}\right)^{2}}{\rho}\right] \\
& -D_{B}^{*}\left[\frac{\rho_{A}}{\rho} \Delta \rho_{B}+\frac{\vec{\nabla} \rho_{A} \cdot \vec{\nabla} \rho_{B}}{\rho}\right] \\
\frac{\partial \rho_{B}}{\partial t}= & k_{-3}-k_{3} \frac{\rho_{B}}{\rho}\left(\rho-\rho_{A}-\rho_{B}\right)-k_{2}\left(\rho_{A}\right)^{2} \rho_{B} \\
& -D_{A}^{*}\left[\frac{\rho_{B}}{\rho} \Delta \rho_{A}+\frac{\vec{\nabla} \rho_{A} \cdot \vec{\nabla} \rho_{B}}{\rho}\right] \\
& +D_{B}^{*}\left[\left(1-\frac{\rho_{B}}{\rho}\right) \Delta \rho_{B}-\frac{\left(\vec{\nabla} \rho_{B}\right)^{2}}{\rho}\right]
\end{aligned}
$$


where $\Delta=\vec{\nabla}^{2}$ denotes the Laplacian. In a 1-dimensional system, we have $\Delta=\frac{\partial^{2}}{\partial x^{2}}$ and $\nabla=\frac{\partial}{\partial x}$. Hence, in a solution of species $\mathrm{A}$ and $\mathrm{B}$ that cannot be considered dilute, the transport by diffusion of a given species depends on the densities, $\rho_{A}, \rho_{B}$, and the diffusion coefficients, $D_{A}^{*}, D_{B}^{*}$, of the two species. It is worth noting that Eqs. $(4,5)$ associated with the unperturbed reaction-diffusion system in dilute conditions are retrieved from Eqs. $(14,15)$ in the limit where $\rho_{A} / \rho$ and $\rho_{B} / \rho$ tend to zero.

\section{NUMERICAL INTEGRATION PROCEDURE}

In order to numerically solve the reaction-diffusion equations, a discrete space variable, $i=x / \Delta x$, and a discrete time variable, $s=t / \Delta t$, are introduced, where $\Delta x$ is the length of a spatial cell $i$ and $\Delta t$ is the integration time step. The Euler method is used to integrate Eqs. $(4,5)$ for the ideal solution and Eqs. $(14,15)$ for the non dilute solution. The discretized perturbed equation for species $\mathrm{A}$ is given to illustrate the numerical procedure:

$$
\begin{aligned}
\rho_{A}(i, s+1)= & \rho_{A}(i, s)+\Delta t\left\{-k_{1} \frac{\rho_{A}(i, s)}{\rho}\left(\rho-\rho_{A}(i, s)-\rho_{B}(i, s)\right)\right. \\
& +k_{2} \rho_{A}(i, s)^{2} \rho_{B}(i, s) \\
& +\frac{D_{A}^{*}}{\rho(\Delta x)^{2}}\left[\left(\rho-\rho_{A}(i, s)\right)\left(\rho_{A}(i+1, s)+\rho_{A}(i-1, s)-2 \rho_{A}(i, s)\right)\right. \\
& \left.-\left(\rho_{A}(i+1, s)-\rho_{A}(i, s)\right)^{2}\right] \\
& -\frac{D_{B}^{*}}{\rho(\Delta x)^{2}}\left[\rho_{A}(i, s)\left(\rho_{B}(i+1, s)+\rho_{B}(i-1, s)-2 \rho_{B}(i, s)\right)\right. \\
& \left.\left.+\left(\rho_{A}(i+1, s)-\rho_{A}(i, s)\right)\left(\rho_{B}(i+1, s)-\rho_{B}(i, s)\right)\right]\right\}
\end{aligned}
$$

where $\rho_{I}(i, s)$ is the density of species $\mathrm{I}$ in spatial cell $i$ at discrete time $s$. The parameter values are chosen in the domain of stability of Turing pattern and set at $k_{1}=2.5673, k_{2}=0.8793, k_{3}=1.9255, k_{-3}=7.7019, D_{A}^{*}=2.7, D_{B}^{*}=27[12]$. The rate constant values and the diffusion coefficient values impose the wavelength of the Turing pattern at $\lambda=12.7$, as explained in the appendix B. In order to optimize the accuracy of the numerical results, the length $\Delta x$ of a spatial cell is adjusted in such a way that the wavelength of the unperturbed structure corresponds to a sufficiently large, integer number of cells. Specifically, we choose $\lambda / \Delta x=38$, which imposes $\Delta x=0.33527$. The number of digits does not reveal that the model suffers from a high sensitivity to small variations of the parameters but simply results from requiring that the wavelength exactly spans over an integer number of spatial cells. It is to be noted that we studied the effects of fluctuations on the structure and proved that, instead of blurring it, noise sustains the pattern [10]. The requirements of the numerical integration procedure impose a sufficiently small integration time step, $\Delta t=0.000345$, such that the numerical coefficients in front of the reactive terms and the diffusive terms are smaller than 1 and $\frac{1}{2}$, respectively [58]. If the 
values of the rate constants and diffusion coefficients of well-identified morphogens would be available for a specific vertebrate embryo, the model could be checked by comparing the wavelength of the Turing structure with the actual value of somite size. But such a quantitative analysis far exceeds the scope of this study, in which the length scale and time scale are arbitrary. In the following, lengths are scaled by the length $\Delta x$ of a spatial cell and time is scaled by the integration time step $\Delta t$, so that the accuracy of the discrete integration scheme may be straightforwardly evaluated.

We denote by $\left(\rho_{A}^{h}, \rho_{B}^{h}\right)$, for $h=1,2,3$, the three homogeneous steady states of the unperturbed system and $\left(\rho_{A}^{* h}, \rho_{B}^{* h}\right)$, the three steady states of the crowded system. The initial conditions are analogous in the two situations. Step functions are chosen for the initial density profiles of species $\mathrm{A}$ and $\mathrm{B}$ prepared in $n_{0}=150$ spatial cells for both the unperturbed system and the non dilute system. The 10 first spatial cells, supposed to mimic the vicinity of the head of the embryo, are prepared in the steady state $\left(\rho_{A}^{1}=\left(k_{2} k_{-3}+\sqrt{\left(k_{2} k_{-3}\right)^{2}-4\left(k_{1}\right)^{2} k_{2} k_{3}}\right) /\left(2 k_{1} k_{2}\right), \rho_{B}^{1}=\left(k_{-3}-k_{1} \rho_{A}^{1}\right) / k_{3}\right)$. In agreement with the preexistence of the head, zero-flux boundary conditions are chosen at the rostral end, so that the density profiles of species $\mathrm{A}$ and $\mathrm{B}$ have an extremum at this boundary, once the Turing pattern have developed. The $\left(n_{0}-10\right)$ next spatial cells are prepared in the steady state $\left(\rho_{A}^{3}=0, \rho_{B}^{3}=k_{-3} / k_{3}=4\right)$ for the unperturbed system and in the steady state

$$
\begin{aligned}
\rho_{A}^{* 3} & =0 \\
\rho_{B}^{* 3} & =\frac{\rho}{2}\left(1-\sqrt{1-4 k_{-3} /\left(k_{3} \rho\right)}\right) \simeq 4.143
\end{aligned}
$$

for the crowded system.

Initially, the density of species $\mathrm{A}$ is higher than the density of $\mathrm{B}$ at the rostral end and the contrary is observed at the caudal end. The autocatalytic reaction given in Eq. (2) or Eq. (7) produces A and consumes B so that species A invades and replaces species B. Hence, a travelling front emerges as a solution of the equations and the density of species $\mathrm{B}$ tends to decrease at a given distance from the caudal end. At this extremity, the boundary conditions are different from those chosen in references $[12,13]$ in which unlimited, free growth was considered. The growth of embryos of finite size is obtained as follows. We add a spatial cell at the caudal end at a constant rate, that we choose smaller than the propagation speed of the wave front, imposed by the dynamical parameters. Hence, the density of species $\mathrm{B}$ at a given distance from the caudal end decreases. Such conditions correctly reproduce that the presomitic mesoderm, comprised between the growing caudal end and the faster travelling antagonist gradients of $\mathrm{A}=$ retinoic acid and $\mathrm{B}=\mathrm{Fgf}$, gradually shrinks as observed for many vertebrates, such as zebrafish, chickens, mice and snakes [1].

In order to stop the simulation before the travelling wave reaches the very end of the medium, we introduce a threshold $\epsilon$, such that front propagation and somite 
growth are arrested when the density of species B at a given distance from the caudal extremity falls below this threshold. More precisely, the dilute system continues to grow as long as $\rho_{B}((n-100) \Delta x, t)>\epsilon$. In other words, the numerical resolution is stopped at time $t=t_{\text {stop }}$ for the unperturbed system when the density of species B in the spatial cell $(n-100)$ becomes smaller than the threshold $\epsilon$ for an embryo of total size $n$. Similarly, somite growth in the crowded system is arrested at time $t=t_{\text {stop }}^{*}$ when $\rho_{B}$ crosses the same threshold $\epsilon$ in the spatial cell $\left(n^{*}-100\right)$. Figure 1 illustrates the boundary conditions chosen to mimic the growth of an embryo as long as the threshold $\epsilon$ has not been crossed. Periodic spatial oscillations of the densities $\rho_{I}$ of species $\mathrm{I}=\mathrm{A}, \mathrm{B}$ are formed according to Turing instability which develops behind the propagating wave front. Between the travelling gradients of species A and $\mathrm{B}$ and the caudal end, the unstructured region mimics the presomitic mesoderm. The values of $n$ and $n^{*}$ are chosen on the basis of a trial and error procedure in such a way that the same number of wavelengths, fixed at 21, are formed in the two cases when the numerical resolution stops, i.e. when $\rho_{B}$ crosses $\epsilon$ in the spatial cells $n-100$ and $n^{*}-100$ in the dilute and confined systems, respectively. Using this trick, we are able to assign a length $L=n \Delta x$ to the ideal solution and $L=n^{*} \Delta x$ to the non dilute system. A more refined termination process could be envisaged to form smaller somites at the caudal end, but this point is not the concern of the present study.

As already pointed out, the ideal solution is retrieved in the limit where the solvent is in great excess with respect to the solutes $\mathrm{A}$ and $\mathrm{B}$, i.e. when $\left(\rho_{A}(x, t)+\right.$ $\left.\rho_{B}(x, t)\right) / \rho \rightarrow 0$, with $\rho$ given in Eq. (10). In an inhomogeneous, growing system in which a spatial structure develops, the evaluation of the departure from ideality by a single quantity requires the choice of a location where the system remains in a stationary state. To this goal, we choose the caudal end of the embryo, characterized by the homogeneous steady state $\left(\rho_{A}^{* 3}, \rho_{B}^{* 3}\right)$ given in Eqs. $(17,18)$. Hence, we define the strength of confinement or departure from ideality by the ratio between the sum of the densities of species $\mathrm{A}$ and $\mathrm{B}$ and the total density evaluated at the steady state $\left(\rho_{A}^{* 3}, \rho_{B}^{* 3}\right)$ :

$$
\delta=\frac{\rho_{A}^{* 3}+\rho_{B}^{* 3}}{\rho}
$$

Acording to Eqs. $(17,18)$, it reads:

$$
\delta=\frac{1}{2}-\frac{1}{2} \sqrt{1-4 k_{-3} /\left(k_{3} \rho\right)}
$$

which highlights that the total density $\rho$ may be used as a convenient parameter to control the departure from ideality, without changing the rate constants and the diffusion coefficients, i.e. without affecting the unperturbed equations given in Eqs. $(4,5)$. In particular, Eq. (20) shows that the parameter $\delta$ and the departure from ideality decrease as the total density $\rho$ increases. The ideal solution, where the solvent is in great excess, is associated with the limit $\rho \rightarrow \infty$ for which $\delta \rightarrow 0$. In the next section, we present the differences between the spatial structures obtained in a more or less confined system. 


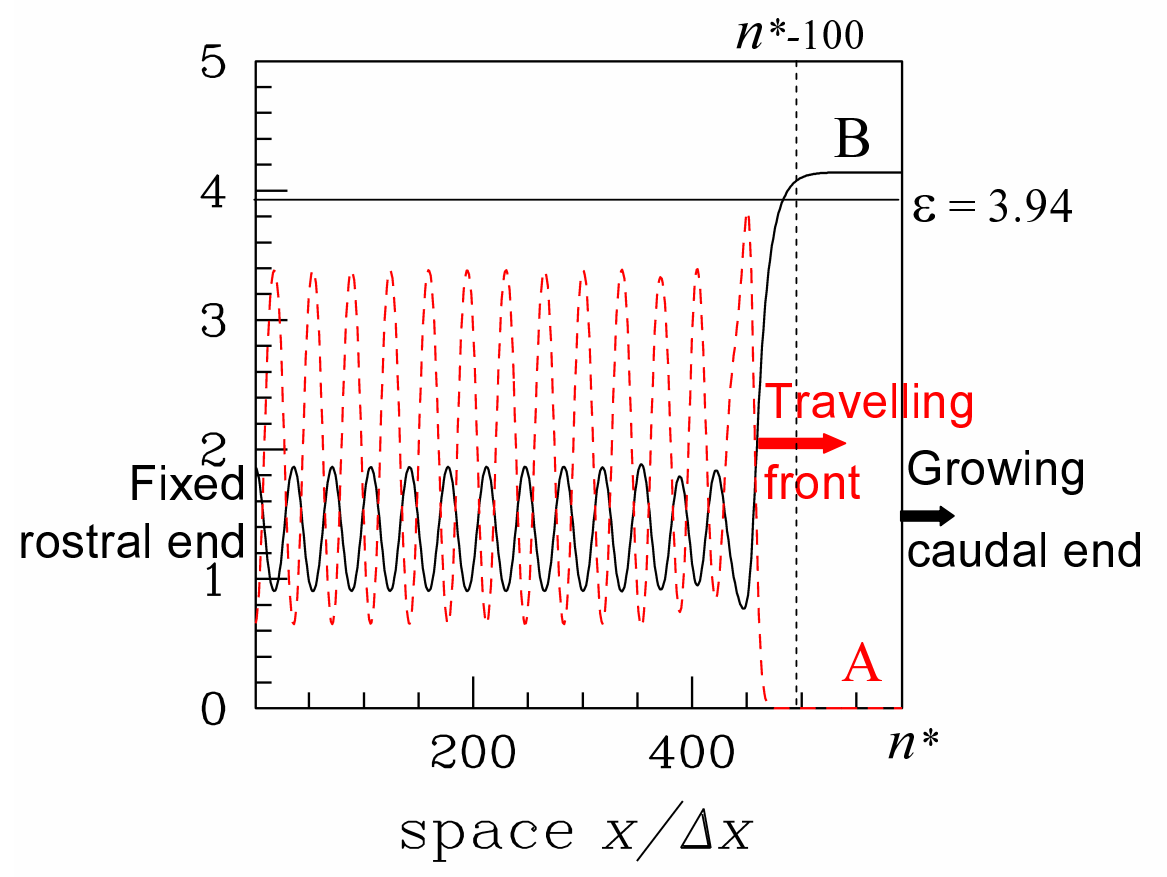

Figure 1: (Color online) Snapshot of the spatial density profiles, $\rho_{A}$ and $\rho_{B}$, of species A (red dashed line) and B (black solid line) at time $t^{*}=350000 \Delta t$, solution of the equations in a crowded system (Eqs. $(14,15))$ before the end of system growth. The parameters take the following values in arbitrary units: $k_{1}=2.5673, k_{2}=0.8793$, $k_{3}=1.9255, k_{-3}=7.7019, D_{A}^{*}=2.7, D_{B}^{*}=27, \Delta t=0.000345, \Delta x=0.33527$, $\rho=120$. The growing caudal end has reached the spatial cell $n^{*}=592$. The travelling gradients of species $\mathrm{A}$ and $\mathrm{B}$ propagate faster than the system grows and the density of species $\mathrm{B}$ at a given distance from the caudal end, $\rho_{B}\left(\left(n^{*}-100\right) \Delta x, t^{*}\right)$, tends to decrease. At time $t^{*}$, the threshold $\epsilon=3.94$ is not yet crossed in the spatial cell $n^{*}-100=492$ and growth is not stopped. 


\section{RESULTS}

We look for numerical solutions of the unperturbed equations (Eqs. $(4,5))$ in a dilute solution and the modified equations (Eqs. $(14,15)$ ) for a crowded system.

The results are given in Fig. 2 for the unperturbed system associated with $\delta=0$ and a small, confined system associated with $\delta=0.0345$ according Eq. (20) for the parameter values given in Fig. 1. At a given time, the embryo is supposed to be oriented with the head on the left and the caudal end on the right of the figure: In both the unperturbed and confined systems, a stationary, periodic spatial pattern of Turing type develops behind a propagating wave front. The periodic spatial oscillations between green and blue colors reveal the succession of minima and maxima of the density $\rho_{B}$ of species $\mathrm{B}$ in the structured region, red region can be identified with the unstructured caudal tissue, and yellow region is outside the embryo. The same threshold, $\epsilon=3.94$, is reached earlier in the case of the confined system and the length $L$ of the system is smaller. In the following, we discuss the consequences of confinement on wave front propagation speed and morphogen gradient, growth rate (i.e. embryo size), and wavelength of the structure (i.e. somite size).

\subsection{Effect of confinement on wave front propagation speed and morphogen gradient}

The propagation speed of the chemical wave front is given by the slope of the blue line between the green and blue periodic pattern and the red unstructured region in Fig. 2. We find a wave front propagation speed of $v_{p}=0.934 \times 10^{-3} \Delta x / \Delta t$ for the unperturbed system and $v_{p}^{*}=1.33 \times 10^{-3} \Delta x / \Delta t$ for the confined system. One of the effects of confinement in this model is thus to speed up somite formation process behind the propagating front. Limited experimental evidence of the correlation between growth speed and embryo size is reported in the literature. Experiments on mouse embryos whose size has been reduced by a treatment with mitomycin $\mathrm{C}$ reveal a complex, non monotonous variation of growth speed: treated and consequently smaller embryos show an early phase of growth retardation but accelerate their growth after 9.5 days post coitum (p.c.) and actually grow faster than normal embryos after 10 days p.c. [59]. However, the correlation between morphogen gradient size and embryo size has been repeatedly observed for both vertebrates and invertebrates $[2,4,5,7]$.

The morphogen gradient is defined as the slope of the morphogen density profile at the inflection point of the travelling wave front, located between the Turing pattern and the steady state $\left(\rho_{A}^{* 3}, \rho_{B}^{* 3}\right)$. The final density profiles of the two species $\mathrm{A}$ and $\mathrm{B}$, supposed to play the role of morphogens, are given in Fig. 3 in the case of an unperturbed system and a crowded one. The density profiles are used to evaluate the morphogen gradients. For species $\mathrm{B}$, the morphogen gradient is found to increase from $g_{B}=0.16 / \Delta x$ for the unperturbed system to $g_{B}^{*}=0.18 / \Delta x$ for the confined system. Similar results are obtained for species A. Hence, the increase of 


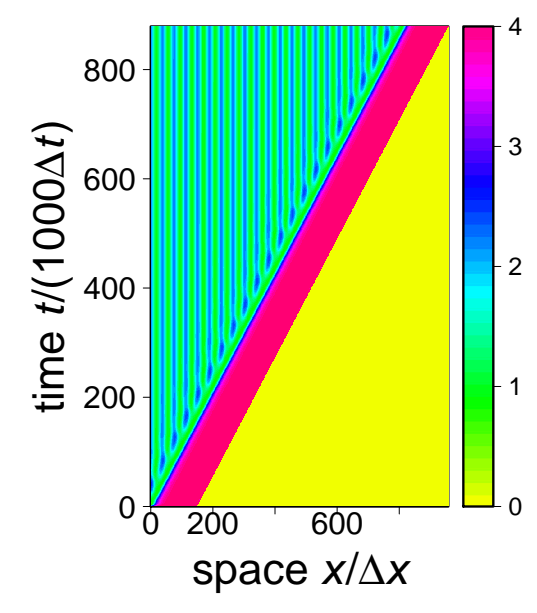

(a)

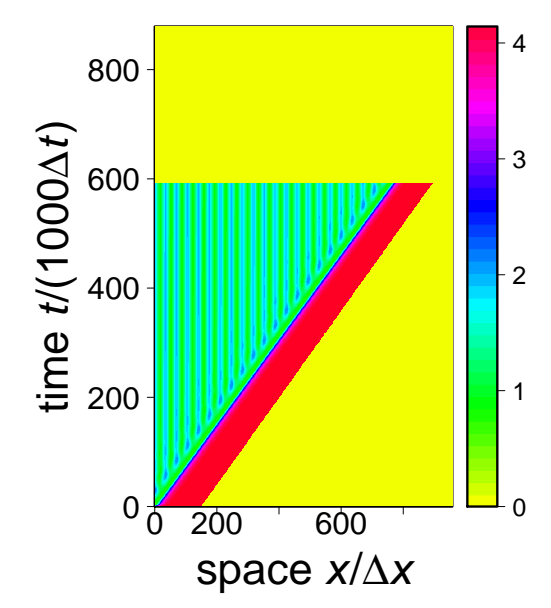

(b)

Figure 2: (Color online) Time-space evolution of the density $\rho_{B}(x, t)$ of $\mathrm{B}$ species (see color scale), (a) solution of the unperturbed equations (Eqs. (4,5)) and (b) solution of the equations in a crowded system (Eqs. $(14,15))$. The parameter values are given in the caption of Fig. 1. The threshold, $\epsilon=3.94$, is reached in spatial cell $n-100=868$ at time $t_{\text {stop }}=878264 \Delta t$ in the unperturbed system and in spatial cell $n^{*}-100=795$ at time $t_{\text {stop }}^{*}=591836 \Delta t$ in the confined system. The yellow regions are outside the simulated system. 

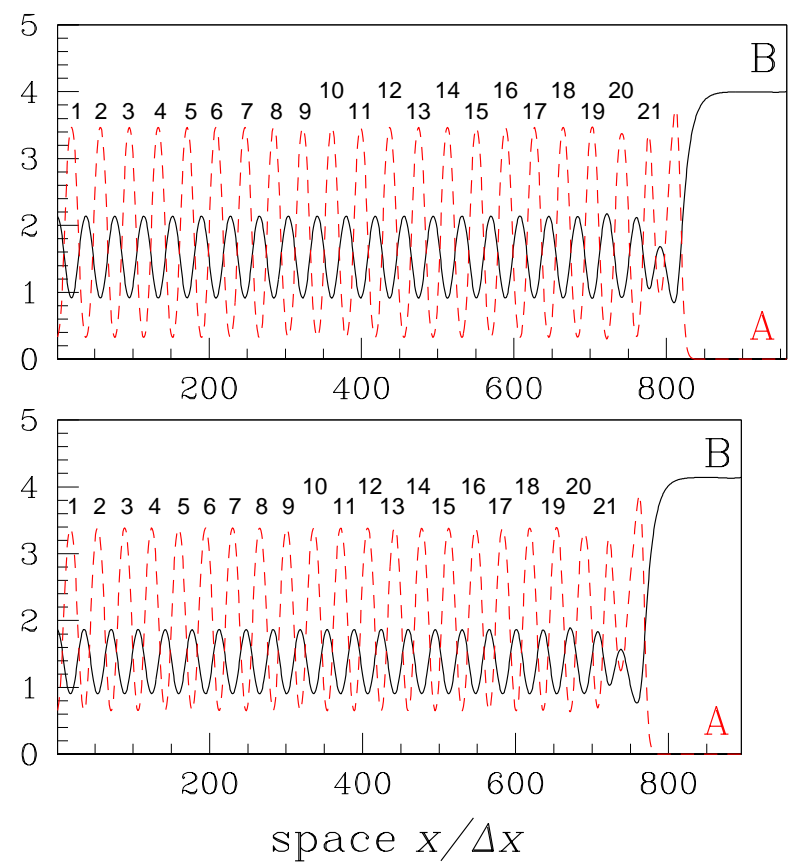

Figure 3: (Color online) Final spatial density profiles, $\rho_{A}$ and $\rho_{B}$, of species A (red dashed line) and B (black solid line), solutions of the unperturbed equations (top, Eqs. $(4,5))$ at time $t_{\text {stop }}=878264 \Delta t$ and solutions of the equations in a crowded system (bottom, Eqs. $(14,15)$ ) at time $t_{\text {stop }}^{*}=591836 \Delta t$. The parameter values are given in the caption of Fig. 1. 
the front propagation speed is directly correlated with the increase of the travelling gradients of species A and B when the solution departs from ideality. Hence, the model predicts that an embryo, suffering from a strengthening of confinement and of consequently smaller size, is associated with a wave front of steeper gradient, in agreement with experiments on vertebrates [2] and invertebrates $[4,7]$.

\subsection{Effect of confinement on system length}

We first examine the growth speed of the embryo in the two limiting cases presented in Figs. 2, 3 in the specific case where the animal is supposed to have 21 somites. In Fig. 2, the slope of the limit between the red presomitic mesoderm and the yellow region, outside the embryo, represents the growth speed of the embryo, denoted by $v_{g}$ and $v_{g}^{*}$ for the unperturbed and confined systems, respectively. We recall that the termination of the spine formation process is reproduced first, by imposing a smaller growth speed of the embryo than the front propagation speed, and secondly, by arresting somite formation as the density of species B in the spatial cell $(n-100)$ or $\left(n^{*}-100\right)$ falls below the threshold $\epsilon$. In both cases, we have $v_{g}<v_{p}$ and $v_{g}^{*}<v_{p}^{*}$. Specifically, the speed $v_{g}=0.920 \times 10^{-3} \Delta x / \Delta t$, at which the unperturbed system grows, is chosen to obtain 21 somites when the density $\rho_{B}((n-100) \Delta x, t)$ crosses the chosen threshold value $\epsilon=3.94$, i.e. when growth is arrested. Then, the growth rate of the presomitic mesoderm in the non dilute system is adjusted in such a way that the same threshold $\epsilon$ is crossed for $\rho_{B}$ in cell $n^{*}-100$ when the same number of somites has been formed. It reads: $v_{g}^{*}=1.27 \times 10^{-3} \Delta x / \Delta t$. We find that the simulation stops when the embryo reaches the length $L=968 \Delta x$ in the unperturbed case and $L^{*}=895 \Delta x$ in the confined system. In the two cases, 21 wavelengths supposed to correspond to the formation of 21 somites are observed, but, in a confined environment, the larger propagation speed $v_{p}^{*}$ of the wave front induces the faster growth of the embryo which reaches earlier the definitive number of somites for a smaller total length.

We then perform a systematic analysis of the variation of system length $L$ with the departure from ideality evaluated by the parameter $\delta$ defined in Eqs. $(19,20)$. Specifically, the equations associated with a crowded system (Eqs. $(14,15)$ ) are numerically solved for the same parameter values except the total density $\rho$. Equation (20) is used to compute the departure from ideality $\delta$. The total number of cells $n^{*}$ which fixes the length $L=n^{*} \Delta x$ of the system associated with different values of $\delta$ is determined by trial and error until 21 somites are formed when the threshold $\epsilon$ is reached in cell $n^{*}-100$. The results are given in Fig. 4. Although the approach is deterministic, the results look noisy because of the procedure used to stop the numerical solution. With a finer spatial discretization, i.e. more cells per wavelength, a better accuracy on the system length $L$ would be obtained. As expected, the behavior of the dilute solution obtained by solving the unperturbed equations (Eqs. $(4,5)$ ) is recovered when solving the equations for a crowded system for a sufficiently small value of $\delta$. At the precision of the numerical solutions, the unperturbed results are retrieved for $\delta=0.0004$. When the departure from ideality 


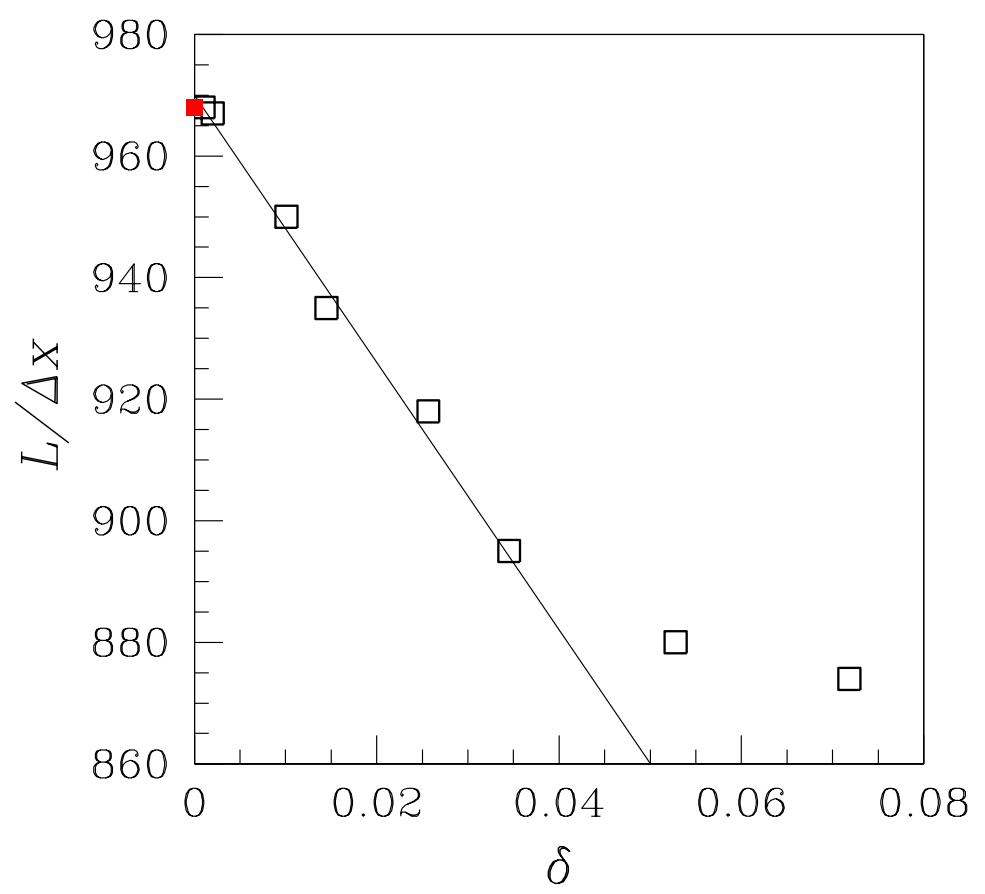

Figure 4: (Color online) Dimensionless system length $L / \Delta x$ versus departure from ideality $\delta=\frac{\rho_{A}^{* 3}+\rho_{B}^{* 3}}{\rho}$. The open squares are obtained by solving Eqs. $(14,15)$ for a crowded system for the parameter values given in the caption of Fig. 1 and a variable total density $\rho$. The line is a linear fit of the data in the domain $\delta \in[0,0.035]$. The red solid square gives the results of Eqs. $(4,5)$ for the unperturbed system. 
is large, for $\delta>0.035$, the decrease of system length is nonlinear. For such strong perturbations, the hypotheses leading to Eqs. (A.2,A.9) are not supposed to be valid. Interestingly, a linear relationship between system length $L$ and the strength of confinement is obtained in the interval $\delta \in[0,0.035]$, which defines the domain of validity of the approach. An embryo of normal size $L$ is supposed to be associated with a departure $\delta$ from ideality inside the interval $[0,0.035]$. In order to optimize the difference between the system sizes observed in Figs. 2, 3, we have chosen to show the results obtained for the boundaries of the domain of validity, i.e. the limit $\delta \rightarrow 0$ of a dilute solution, which leads to the largest embryo, and the nonideal case $\delta=0.035$, which leads to a small embryo.

\subsection{Effect of confinement on the wavelength of the pattern}

In the structured region shown in Figs. 2, 3, the maxima of $\rho_{B}$ are supposed to initiate the formation of boundaries between somites so that somite size may be evaluated by the wavelength of the spatial structure. As shown by the final density profiles in Fig. 3, we find that the wavelength $\lambda^{*} \simeq 35.1 \Delta x$ in the crowded system is smaller than the unperturbed value $\lambda=38 \Delta x$. Hence, confinement in the smaller, non dilute system leads to the formation of somites of smaller size than in the unperturbed system. The connection between the departure from ideality $\delta$ and system size $L$ has been proven in Fig. 4. Figure 5 shows the correlation between system size $L$ and the wavelength $\lambda^{*}$ of the structure. The linear relationship between $L$ and $\lambda^{*}$ clearly reproduces the scaling behavior observed between embryo size and somite size. The main result of the paper is that the departure from ideality in a smaller system induces the decrease of the wavelength of a Turing structure. Confinement can be reasonably considered as a phenomenon participating in the adaptation of somite size to system size in the framework of a Turing pattern.

An analytical evaluation of the wavelength in the confined system is performed in the appendix B. In particular, we show that the perturbation of diffusion due to confinement has a smaller impact on the spatial structure properties than the perturbation of the chemical reactions. This result is in line with experimental measurements of diffusion coefficients in different dipteran species. Contrary to the morphogen gradient, effective diffusion is found to be essentially the same for embryos of very different sizes [5].

Consequently, we derive the dispersion relations for the confined system with unperturbed diffusion. The selected wavelength of the structure is associated with the mode for which the dispersion relation is maximum. The results are given in Fig. 6 . The selected mode $q_{\text {max }}^{c}$ in the confined system with unperturbed diffusion is larger than the selected mode $q_{\max }$ in the unperturbed system. The selected wavelength, $\lambda^{c}=2 \pi / q_{\max }^{c} \simeq 35.3 \Delta x$, in the system with perturbed reactions is smaller than the unperturbed wavelength $\lambda=2 \pi / q_{\max } \simeq 38 \Delta x$. The value of $\lambda^{c}$ compares well with the numerical value $\lambda^{*} \simeq 35.1 \Delta x$ observed in Fig. 3 when solving Eqs. $(14,15)$, in which both reactions and diffusion are perturbed by confinement. 


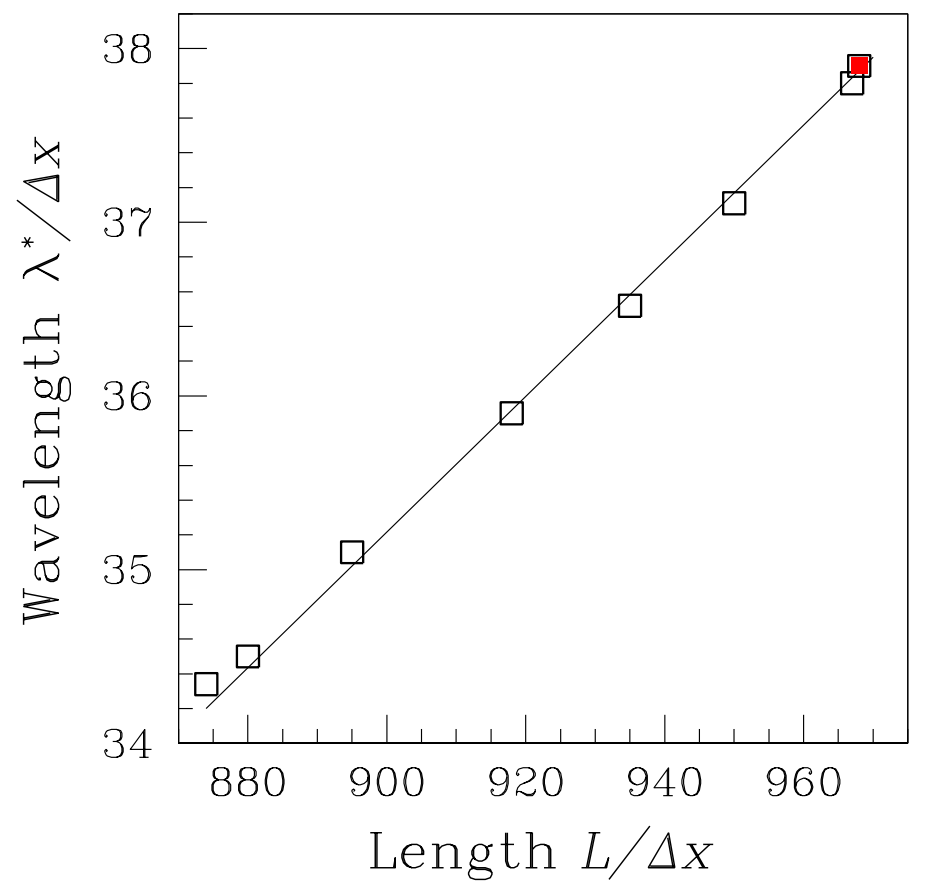

Figure 5: (Color online) Dimensionless wavelength $\lambda^{*} / \Delta x$ of the pattern versus dimensionless system length $L / \Delta x$. The open squares are obtained by solving Eqs. $(14,15)$ for a crowded system for the parameter values given in the caption of Fig. 1 and a variable total density $\rho$ associated with a departure from ideality in the interval $\delta \in[0,0.035]$. The red solid square is obtained by solving Eqs. $(4,5)$ for the dilute system. The line is a linear fit of the data. 


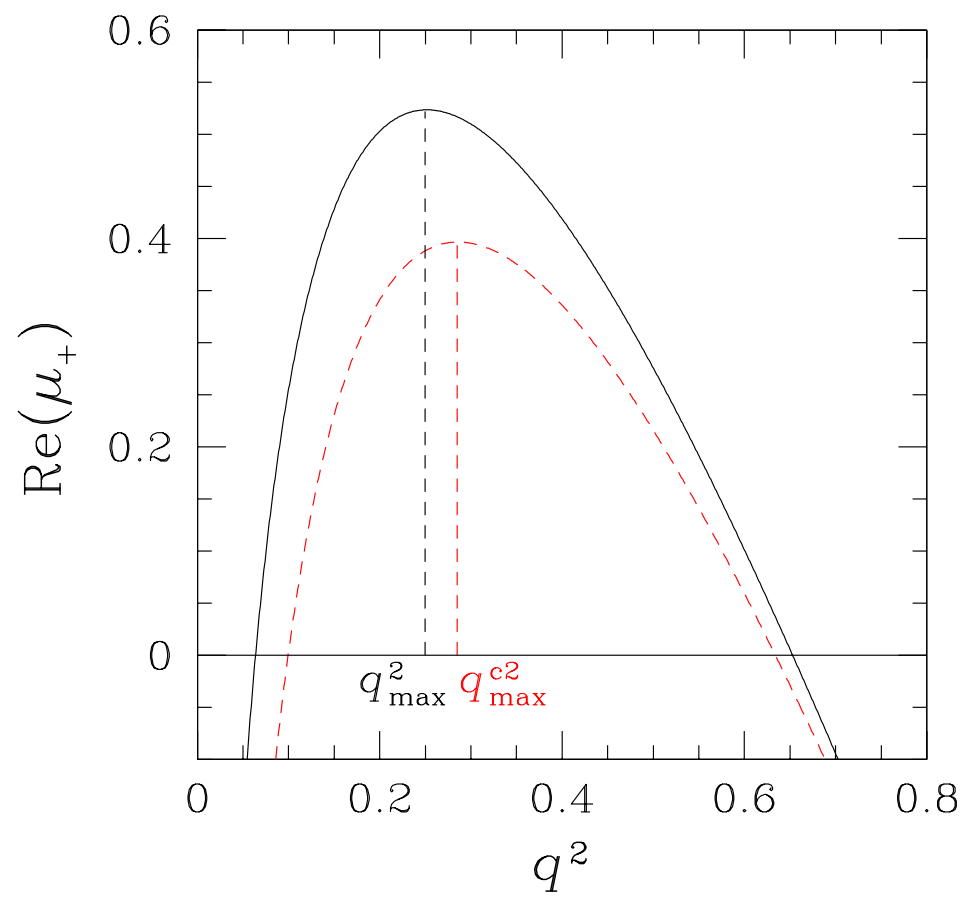

Figure 6: (Color online) Dispersion relations. Real part of the eigenvalues of the linear operator of Eqs. (4,5) associated with the unperturbed system (black solid line) around the steady state $\left(\rho_{A}^{1} . \rho_{B}^{1}\right)$ versus square of the mode $q^{2}$. The parameter values are given in the caption of Fig. 1 . The selected wavelength is deduced from the maximum of the curve: $\lambda=2 \pi / q_{\max }$. Analogous results for the perturbed chemical scheme given in Eqs. (6-8) and unperturbed diffusion (red dashed line) around $\left(\rho_{A}^{c 1}, \rho_{B}^{c 1}\right)$. The selected wavelength, $\lambda^{c}=2 \pi / q_{\max }^{c}$, is smaller than $\lambda$ and compares well with the numerical value $\lambda^{*}$ observed in Fig. 3 when solving numerically Eqs. $(14,15)$.

Hence, a reaction-diffusion model, which takes into account a departure from dilute conditions, is able to reproduce that a smaller embryo faster presents a correct number of somites of smaller size. The larger propagation speed of the wave front observed as confinement increases is associated with a steeper concentration gradient [2]. The results can be straightforwardly extended to the segmentation of invertebrates. In agreement with experiments on Drosophila, our model based on Turing pattern correlates the scaling of patterning with the modification of the steepness of the signaling gradient $[4,7]$. 


\section{CONCLUSION}

In this paper, we consider a reaction-diffusion model of somitogenesis based on the mechanism of Turing instability and are dealing with the main concern of this kind of model, which is its ability to account for pattern size adaptation to total embryo size. We assume that in a smaller embryo, molecular crowding is reinforced, so that non specific interactions of the reactive species with the solvent cannot be ignored in the reaction scheme and in the transport by diffusion.

We derive a modified Fick's first law in the framework of linear irreversible thermodynamics and write perturbed reaction-diffusion equations, valid in a non dilute system. We find that confinement leads to a steeper travelling gradient of morphogen, a larger propagation speed of the chemical wave front and consequently to the faster formation of the total number of somites, but of smaller size. Hence, we have developed a model based on Turing pattern which incorporates the effects of crowding and predicts the formation of smaller somites in smaller embryos, in agreement with the experimental observations.

Our results prove that Turing modeling of somitogenesis cannot be discarded by invoking the question of scaling in embryonic development. Moreover, the reactiondiffusion model we propose has the advantage to be based on elementary microscopic processes and to display some universal features, in so far as it may be used to model spine formation as well as segmentation of invertebrates. Our approach sheds a new light on the role that the solvent can play in biological phenomena, in which it is often disregarded.

\section{A APPENDIX A}

This appendix is devoted to the derivation of the modified laws of diffusion in a non dilute solution. The linear irreversible thermodynamics framework can be used to establish Fick's laws in a ternary mixture of A, B and $*$ [42]. The entropy production per unit mass due to isothermal diffusion is usually given in the framework of the center of mass of the solution:

$$
\sigma=\frac{1}{T} \sum_{I=A, B, *} \vec{j}_{I} \cdot\left(-\vec{\nabla}_{T} \mu_{I}\right)
$$

where $\vec{\nabla}_{T}$ denotes the spatial gradient at constant temperature $T$ and $\mu_{I}$, the chemical potential of species I. The flux $\vec{j}_{I}$ of species I is said to be conjugated to the opposite of the gradient of the chemical potential of I. Fick's first law is a straightforward consequence of linear relationships between the fluxes of species $\mathrm{A}$ and $\mathrm{B}$ and the conjugated forces $\left(-\vec{\nabla}_{T} \mu_{A}\right)$ and $\left(-\vec{\nabla}_{T} \mu_{B}\right)$.

Specifically, the effect of confinement is supposed to be sufficiently small to ensure that the chemical potential $\mu_{I}$ of species I per unit mass is the same as in an ideal 
solution:

$$
\mu_{I}=\mu_{I}^{0}+\frac{R T}{M_{I}} \ln \rho_{I}
$$

where $\mu_{I}^{0}$ is the standard chemical potential of species I per unit mass, $R$, the gas constant, and $M_{I}$, the molar mass of species I.

The framework of the solvent $*$ is more convenient to establish Fick's laws in a crowded environment. The flux of species I in the framework of the solvent is defined by $\vec{j}_{I}^{*}=\rho_{I}\left(\vec{u}_{I}-\vec{u}_{*}\right)$ where $\vec{u}_{*}$ is the velocity of the solvent. By definition, the flux of the solvent vanishes in the framework of the solvent:

$$
\overrightarrow{j_{*}^{*}}=0 .
$$

In the case of isothermal diffusion in a non viscous fluid, mechanical equilibrium at constant $\rho$ leads to $\vec{\nabla} p=0$ and Gibbs-Duhem equation to $\vec{\nabla} p=\sum_{I=A, B, *} \rho_{I} \vec{\nabla}_{T} \mu_{I}$, where $p$ is pressure [42]. Hence, we have:

$$
\sum_{I=A, B, *} \rho_{I}\left(-\vec{\nabla}_{T} \mu_{I}\right)=0
$$

The expression of entropy production given in Eq. (A.1) can be rewritten as:

$$
\begin{aligned}
\sigma & =\frac{1}{T} \sum_{I=A, B, *} \rho_{I}\left(\vec{u}_{I}-\vec{u}_{*}+\vec{u}_{*}-\vec{u}\right)\left(-\vec{\nabla}_{T} \mu_{I}\right) \\
& =\sum_{I=A, B} \vec{j}_{I}^{*}\left(-\vec{\nabla}_{T} \mu_{I}\right)
\end{aligned}
$$

where use has been made of Eqs. (A.3,A.4). Phenomenological coefficients $\Omega_{I J}$ are introduced to write linear relationships between thermodynamic fluxes $\overrightarrow{j_{I}^{*}}$ and forces $\left(-\vec{\nabla}_{T} \mu_{J}\right)$ :

$$
\vec{j}_{I}^{*}=\sum_{J=A, B} \Omega_{I J}\left(-\vec{\nabla}_{T} \mu_{J}\right)
$$

leading to Fick's first law

$$
\vec{j}_{I}^{*}=\sum_{J=A, B} D_{I J}^{*}\left(-\vec{\nabla} \rho_{J}\right)
$$

where the relationship between the diffusion coefficients $D_{I J}^{*}$ and the phenomenological coefficients $\Omega_{I J}$ can be easily deduced from Eq. (A.2). In a dilute solution, the diffusion coefficients $D_{I J}^{*}$ are known to be nearly independent of the densities $\rho_{I}$ and to have negligible non diagonal elements, $D_{I J}^{*} \sim 0$ for $I \neq J$. Exactly as we used the expression of the chemical potential of an ideal solution, we assume that the level of confinement is sufficiently low to ensure $D_{I J}^{*}=D_{I}^{*} \delta_{I J}$, where $\delta_{I J}$ is the Kronecker symbol. The Fick's first law becomes:

$$
\overrightarrow{j_{I}^{*}}=D_{I}^{*}\left(-\vec{\nabla} \rho_{I}\right), \quad I=A, B
$$


The fluxes $\vec{j}_{I}^{*},(I=A, B)$, in the framework of the solvent are related to the fluxes $\vec{j}_{I},(I=A, B, *)$ in the framework of the center of mass by:

$$
\begin{aligned}
\vec{j}_{I}^{*} & =\rho_{I}\left(\vec{u}_{I}-\vec{u}\right)+\rho_{I}\left(\vec{u}-\vec{u}_{*}\right) \\
& =\vec{j}_{I}-\frac{\rho_{I}}{\rho_{*}} \vec{j}_{*}
\end{aligned}
$$

The reverse relations are given by:

$$
\begin{aligned}
& \vec{j}_{A}=\left(1-\frac{\rho_{A}}{\rho}\right) \vec{j}_{A}^{*}-\frac{\rho_{A}}{\rho} \vec{j}_{B}^{*} \\
& \vec{j}_{B}=-\frac{\rho_{B}}{\rho} \vec{j}_{A}^{*}+\left(1-\frac{\rho_{B}}{\rho}\right) \vec{j}_{B}^{*}
\end{aligned}
$$

where Eqs. $(10,12)$ have been used to eliminate $\rho_{*}$ and $\vec{j}_{*}$. Hence, in the framework of the center of mass, the Fick's first law given in Eq. (A.8) leads to:

$$
\begin{aligned}
& \vec{j}_{A}=-\left(1-\frac{\rho_{A}}{\rho}\right) D_{A}^{*} \vec{\nabla} \rho_{A}+\frac{\rho_{A}}{\rho} D_{B}^{*} \vec{\nabla} \rho_{B} \\
& \vec{j}_{B}=\frac{\rho_{B}}{\rho} D_{A}^{*} \vec{\nabla} \rho_{A}-\left(1-\frac{\rho_{B}}{\rho}\right) D_{B}^{*} \vec{\nabla} \rho_{B}
\end{aligned}
$$

These equations are used to derive the divergence of the fluxes, $\vec{\nabla} \cdot \vec{j}_{I}$, in the balance equations (Eq. (9)) which govern the dynamics of the densities $\rho_{I}$ for $I=A, B$ in a non dilute solution.

\section{B APPENDIX B}

In order to evaluate the wavelength of the spatial structure in a confined environment, we first begin with the comparison of different quantities numerically obtained when considering that confinement only modifies either diffusion or reaction.

Hence, we start with Eqs. $(14,15)$ and replace the reaction terms by the one of the unperturbed system given in Eqs. $(4,5)$. For the system in which only diffusion is supposed to be modified by confinement, we obtain the following values of the stationary states: $\left(\rho_{A}^{d 1}=1.843\right) \simeq\left(\rho_{A}^{1}=1.848\right),\left(\rho_{B}^{d 1}=1.542\right) \simeq\left(\rho_{B}^{1}=1.537\right)$, and $\left(\rho_{B}^{d 3}=4.000\right) \simeq\left(\rho_{B}^{3}=4\right)$. The growth rate of the presomitic mesoderm is $v_{g}^{d}=9.70 \times 10^{-4} \Delta x / \Delta t$, and the propagation speed of the chemical wave front is $v_{p}^{d}=9.81 \times 10^{-4} \Delta x / \Delta t$, where the exponent $d$ stands for perturbed diffusion. These different values are rather close to the unperturbed results, but nevertheless prove that the perturbation of diffusion has the tendency to increase wave front propagation speed.

When we numerically solve Eqs. (14,15) with unperturbed diffusion we obtain: $\rho_{A}^{r 1}=2.060, \rho_{B}^{r 1}=1.376$, and $\rho_{B}^{r 3}=4.143 . \quad v_{g}^{r}=1.20 \times 10^{-3} \Delta x / \Delta t$, $v_{p}^{r}=1.265 \times 10^{-3} \Delta x / \Delta t$, where the exponent $r$ stands for perturbed reaction. These results agree with the values $\rho_{A}^{* 1}=2.050, \rho_{B}^{* 1}=1.380$, and $\rho_{B}^{* 3}=4.143$. obtained when numerically integrating Eqs. $(14,15)$ associated with the system in which confinement is supposed to modify both diffusion and reaction. We conclude 
that the perturbation of diffusion by confinement has the same qualitative impact on the spatial structure formation as the perturbation of reaction, but with a smaller amplitude.

Consequently, we may neglect the perturbation of diffusion to evaluate the wavelength of the structure. Hence, we consider Eq. $(14,15)$ with unperturbed diffusion. The Fourier transforms, $A_{q}(t)=\int_{-\infty}^{\infty} \rho_{A}(x, t) \mathrm{e}^{-i q x} \mathrm{~d} x$ and $B_{q}(t)=\int_{-\infty}^{\infty} \rho_{B}(x, t) \mathrm{e}^{-i q x} \mathrm{~d} x$, of the densities are introduced to analyze the linear stability of the homogeneous steady state $\rho_{A}^{r 1}, \rho_{B}^{r 1}$ with respect to inhomogeneous perturbations of wave number $q[60]$. The linear stability operator, $\mathbf{M}^{\mathbf{c}}$, is given by:

$$
\mathbf{M}^{\mathbf{c}}=\left(\begin{array}{cc}
M_{11}=k_{1}\left(1-\rho_{B}^{r 1} / \rho\right)-D_{A}^{*} q^{2} & M_{12}=k_{1} \rho_{A}^{r 1} / \rho+k_{2}\left(\rho_{A}^{r 1}\right)^{2} \\
M_{21}=k_{3} \rho_{B}^{r 1} / \rho-2 k_{1}\left(1-\rho_{A}^{r 1} / \rho-\rho_{B}^{r 1} / \rho\right) & M_{22}=-k_{-3} / \rho_{B}^{r 1}+k_{3} \rho_{B}^{r 1} / \rho-D_{B}^{*} q^{2}
\end{array}\right) \text { B.1) }
$$

The eigenvalues $\mu_{ \pm}$of $\mathbf{M}^{\mathbf{c}}$ are:

$$
\mu_{ \pm}=\left(M_{11}+M_{22} \pm \sqrt{\left(M_{11}+M_{22}\right)^{2}-4\left(M_{11} M_{22}-M_{12} M_{22}\right)}\right) / 2
$$

An analogous procedure is followed to find the eigenvalues of the matrix $\mathbf{M}$ associated with the unperturbed system (Eqs. $(4,5)$ ) [13]. The two dispersion relations for the unperturbed and confined systems are compared in Fig. 6.

In addition to the increase of the mode $q_{\max }^{c}$ in the confined system with respect to the unperturbed value, $q_{\max }$, the maximum value of the real part of the eigenvalue $\operatorname{Re}\left(\mu_{+}\right)$is found smaller. This last result shows that confinement has the tendency to destabilize Turing pattern for the benefit of the homogeneous steady state, which is corroborated by the decrease in the oscillation amplitude of the spatial structure $\left(\Delta \rho_{A}^{*}=2.734\right)<\left(\Delta \rho_{A}=3.144\right)$ and $\left(\Delta \rho_{B}^{*}=0.960\right)<\left(\Delta \rho_{B}=1.224\right)$ observed in Fig. 3.

\section{References}

[1] C. Gomez, E. M. Ozbudak, J. Wunderlich, D. Baumann, J. Lewis, and 0. Pourquié, Nature 454, 335 (2008).

[2] D. Ben-Zvi, B. Z. Shilo, A. Fainsod, A., and N. Barkai, Nature 453, 1205 (2008).

[3] V. M. Lauschke, C. D. Tsiairis, P. François, and A. Aulehla, Nature 493, 101 (2013).

[4] S. J. Day and P. A. Lawrence, Development 127, 2977 (2000).

[5] T. Gregor, W. Bialek, R. R. de Ruyter van Steveninck, D. W. Tank, and E. F. Wieschaus, Proc. Natl Acad. Sci. USA 102, 18403 (2005).

[6] S. Restrepo and K. Basler, Curr. Biol. 21, R815 (2011).

[7] D. Cheung, C. Miles, M. Kreitman, and J. Ma, Development 141, 124 (2014). 
[8] H. Teimouri and A. B. Kolomeisky, J. Chem. Phys. 140, 085102 (2014).

[9] B. Bozorgui, H. Teimouri, and A. B. Kolomeisky, J. Chem. Phys. 143, 025102 (2015).

[10] A. Lemarchand and B. Nowakowski, EPL 94, 48004 (2011).

[11] P. Dziekan, A. Lemarchand, and B. Nowakowski, J. Chem. Phys. 137, 074107 (2012).

[12] P. Dziekan, L. Signon, B. Nowakowski, and A. Lemarchand, J. Chem. Phys. 139, 114107 (2013).

[13] P. Dziekan, L. Signon, B. Nowakowski, and A. Lemarchand, Commun. Theor. Phys. 62, 622 (2014).

[14] A. J. Terry, M. Sturrock, J. K. Dale, M. Maroto, and M. A. J. Chaplain, PLoS One 6, e16980 (2011).

[15] J. Cooke and E. C. Zeeman, J. Theor. Biol. 58, 455 (1976).

[16] R. E. Baker, S. Schnell, and P. K. Maini, Developmental Biology 293, 116 (2006).

[17] M.-L. Déquéant and O. Pourquié, Nature Reviews Genetics 9, 370 (2008).

[18] A. Goldbeter and O. Pourquié, J. Theor. Biol. 252, 574 (2008).

[19] J. Lewis, Y. J. Jiang, B. L. Aerne, L. Smithers, C. Haddon, and D. Ish-Horowicz, Nature 408, 475 (2000).

[20] A. S. Dias, A. S., I. de Almeida, J. M. Belmonte, J. A. Glazier, and C. D. Stern, Science 343, 791 (2014).

[21] A. M. Turing, Philos. Trans. R. Soc. London, Ser. B 237, 37 (1952).

[22] H. Meinhardt and A. Gierer, BioEssays 22, 753 (2000).

[23] Y. Schiffmann, Prog. Biophys. Mol. Biol. 84, 61 (2004).

[24] R. E. Baker, S. Schnell, and P. K. Maini, Int. J. Dev. Biol. 53, 783 (2009).

[25] S. Kondo and T. Miura, Science 329, 1616 (2010).

[26] J. B. A. Green and J. Sharpe, Development 142, 1203 (2015).

[27] A. Gonzalez and R. Kageyama, Gene Regul. Syst. Bio. 1, 35 (2007).

[28] J. D. Murray, Mathematical Biology, I. An Introduction; Springer: New York, 2002.

[29] H. G. Othmer and E. Pate, Proc. Natl Acad. Sci. USA 77, 4180 (1980). 
[30] A. Hunding and P. G. Sorensen, J. Math. Biol. 26, 27 (1988).

[31] S. Ishihara and K. Kaneko, J. Theor. Biol. 238, 683 (2006).

[32] S. Werner, T. Stückemann, M. Beiran Amigo, J. C. Rink, F. Jülicher, and B. M. Friedrich, Phys. Rev. Lett. 114, 138101 (2015).

[33] A. P. Minton, J. Biol. Chem. 276, 10577 (2001).

[34] R. J. Ellis and A. P. Minton, Nature 425, 27 (2003).

[35] J. Sun and H. Weinstein, J. Chem. Phys. 127, 155105 (2007).

[36] Z.-R. Xie, J. Chen, and Y. Wu, J. Chem. Phys. 140, 054112 (2014).

[37] P. M. Kekenes-Huskey, C. Eun, and J. A. McCammon, J. Chem. Phys. 143, 094103 (2015).

[38] P. Nalecz-Jawecki, P. Szymanska, M. Kochanczyk, J. Miekisz, and T. Lipniacki, J. Chem. Phys. 143, 215102 (2015).

[39] P. Ball, Chem. Rev. 108, 74 (2008).

[40] R. Grima and S. Schnell, Biophys. Chem. 124, 1 (2006).

[41] K. Takahashi, S. N. V. Arjunan, and M. Tomita, FEBS Letters 579, 1783 (2005).

[42] S. R. de Groot and P. Mazur, Non-Equilibrium Thermodynamics; North Holland: Amsterdam, 1962.

[43] D. Bullara, Y. De Decker, and R. Lefever, Phys. Rev. E 87, 062923 (2013).

[44] C. Varea, J. L. Aragon, and R. A. Barrio, Phys. Rev. E 56, 1250 (1997).

[45] M. G. Clerc, E. Tirapegui, and M. Trejo, Phys. Rev. Lett. 97, 176102 (2006).

[46] N. Kumar and W. Horsthemke, Phys. Rev. E 83, 036105 (2011).

[47] W.-S. Li, W.-Y. Hu, Y.-C. Pang, T.-R. Liu, W.-R. Zhong, and Y.-Z. Shao, Phys. Rev. E 85, 066132 (2012).

[48] E. P. Zemskov, K. Kassner, M. J. B. Hauser, and W. Horsthemke, Phys. Rev. E 87, 032906 (2013).

[49] J. Schnakenberg, J. Theor. Biol. 81, 389 (1979).

[50] P. Gray and S. K. Scott, Chem. Engng Sci. 39, 1087 (1984).

[51] I. Olivera-Martinez and K. G. Storey, Development 134, 2125 (2007).

[52] Y. J. Jiang, B. L. Aerne, L. Smithers, C. Haddon, D. Ish-Horowicz, and J. Lewis, Nature 408, 475 (2000). 
[53] J. Dubrulle, M. J. McGrew, and O. Pourquié, Cell 106, 219 (2001).

[54] A. F. Schier and W. S. Talbot, Annu. Rev. Genet. 39, 561 (2005).

[55] E. Spiegler, Y. K. Kim, L. Wassef, V. Shete, and L. Quadro, Biochim. Biophys. Acta 1821, 88 (2012).

[56] M. Rhinn and P. Dollé, Development 139, 843 (2012).

[57] G. J. Hausman, D. R. Campion, and F. C. Buonomo, Growth Dev. Aging 55, 43 (1991).

[58] J. Crank, The mathematics of diffusion (Clarendon, Oxford, 1979).

[59] P. P. L. Tam, J. Embryol. Exp. Morph. 65 (Suppl), 103 (1981).

[60] G. Nicolis and I. Prigogine, Self-organization in nonequilibrium systems (Wiley, New York, 1977). 